$\mathrm{P}-\mathrm{\square}$

\title{
Imaging of cisplatin in single cancer cell and visualizing its mechanism of action by FIB-TOF-SIMS
}

\author{
Kazuya Tamura, ${ }^{1}$ Takurou Hasegawa, ${ }^{1}$ Masato Morita, ${ }^{2}$ Kumiko Nagase, ${ }^{3}$ \\ Masatoshi Kakihana, ${ }^{3}$ Naohiro Kajiwara, ${ }^{3}$ Tatsuo Ohira, ${ }^{3}$ Norihiko Ikeda, ${ }^{3}$ Tetsuo Sakamoto ${ }^{1,2, *}$ \\ 1. Graduate School of Electric Engineering and Electronics, Kogakuin University, Tokyo, Japan \\ 2. Department of Applied physics, School of Advanced Engineering, Kogakuin University, Tokyo, Japan \\ 3. Tokyo Medical University Division of Thoracic \& Thyroid Surgery, Tokyo, Japan \\ *corresponding author's e-mail: ct13087@ns.kogakuin.ac.jp
}

(Received: May 20, 2019; Accepted: July 23, 2019)

\begin{abstract}
We examined imaging of cisplatin in single cancer cell and visualization of mechanism of action by time-of-flight secondary ion mass spectrometry (TOF-SIMS). This apparatus has a very high resolution about $40 \mathrm{~nm}$, which was developed ourselves. High intensity signals for PtCN were detected by TOF-SIMS from the cultured cells, which were originated in cisplatin. This $\mathrm{PtCN}$ ion signal was detected from not only the surface of cell but also the intracellular region. Cisplatin is structure where chlorine is replaced with water when combine with the cell nucleus. The results show selectively combine with nucleus. We achieved visualization of distribution of cisplatin intracellular nucleus by TOF-SIMS.
\end{abstract}

\section{Introduction}

Recently, diagnosis and treatment of cancer in medical and pharmaceutical department, and visualization of the effects and distribution of drugs are important. It is necessary to determine an appropriate dose of anticancer drug, because it is especially difficult to judge a proper dose of anticancer drug which have more dander than other drugs. If we test the clinical dose by bioimaging, it is possible to confirm safety and effect of drug in the preclinical stage. Molecular imaging is needed for elucidation of drug delivery systems (DDS) and non-target side effects.

Cisplatin $\left(\mathrm{Pt}\left(\mathrm{NH}_{3}\right)_{2} \mathrm{Cl}_{2}\right)$ is one of the most popular and traditional anticancer drugs. Figure 1 shows the molecular structure of cisplatin. The mechanism of action of cisplatin is thought that it binds intracellular DNA and inhibits replication.

Matrix Assisted Laser Desorption/Ionization (MALDI) is known as an effective imaging technique in bio- and life-sciences. However, lateral resolution of MALDI is limited around a few tens micrometers. This is mainly by the damage region created with the irradiation of laser. Higher resolution can be realized using fluorescence microscope enough for single cell imaging. In compensation for high resolution, fluorescence microscopy cannot identify both inorganic and organic species without labelling reagents. Only two or three reagents can be used simultaneously. This means that comprehensive information is difficult to be obtained.

We have developed a high resolution imaging method by using Ga Focused Ion Beam Time-of-Flight Secondary Ion Mass Spectrometry (FIB-TOF-SIMS) [1]. We used a pulsed FIB of $30 \mathrm{keV} \mathrm{Ga}^{+}$ion. This technique has an ultimate lateral resolution of $40 \mathrm{~nm}$, and micro-machining capability of a sample by the FIB. In this study, we examined to observe the change and behavior of cisplatin intracellular by TOF-SIMS.<smiles>N[PH](N)(Cl)Cl</smiles>

Fig. 1. Molecular structure of cisplatin.

\section{Experiment}

\subsection{Sample preparation}

In this study, the analysis was carried out with a TOF-SIMS apparatus made by our group. The sample 
was cisplatin reagent and cultured cells of human lung cancer cell which is administered $100 \mu \mathrm{M}$ of cisplatin. We cast it to a Si substrate with a dropper, then waiting 1 minute, and removed the supernatant liquid. Then the cultured cells were dried in air. After drying the cells, distilled water was cast to the Si substrate. We repeated this process several times.

\subsection{Analysis by TOF-SIMS}

First, cisplatin reagent was analyzed by TOFSIMS to reveal characteristic mass peaks. Culture cell of human cancer cells which get cisplatin was analyzed in the next. After pre-sputtering as a treatment for analysis, culture cells were sectioned by the FIBmachining and its cross section was analyzed.

\section{Results and discussion}

Figure 2 to 4 show mass spectrometry imaging of cisplatin of reagents and cisplatin in the single culture cell, respectively. In case of cisplatin reagent, as shown in Fig.2, $\mathrm{PtCl}_{2}{ }^{-}$ions were mainly detected. It seems appropriate considering its molecular formal. The cultured cell was sectioned by the FIB-machining and intracellular was analyzed. Figure 3 shows that $\mathrm{PtCN}^{-}$ ions were detected from the nucleus. This signal was detected from not only the surface of cell but also the cell nucleus. In case of cisplatin reagent, PtCN was not detected.

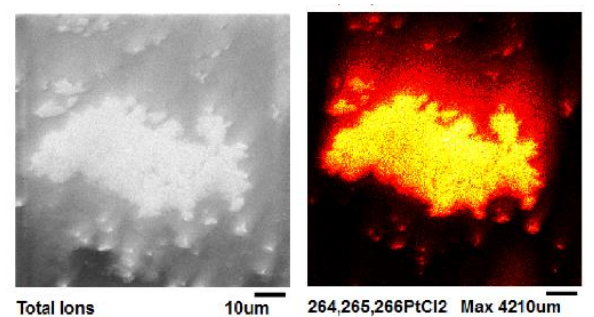

Fig. 2. Imaging of cisplatin of reagents detected.

This result tells us the mechanism of action of cisplatin. Cisplatin is a neutral complex without charge. Chlorine is replaced with water molecules when cisplatin passes into the cell. Therefore, cisplatin is structure which chlorine is replaced with water when combine with cell nucleus. The result, which cisplatin in the cell was detected different structure compare to reagent and selectively combine with nucleus is coincided with mechanism of action of cisplatin, we achieved visualization of distribution of cisplatin intracellular nucleus by TOF-SIMS.
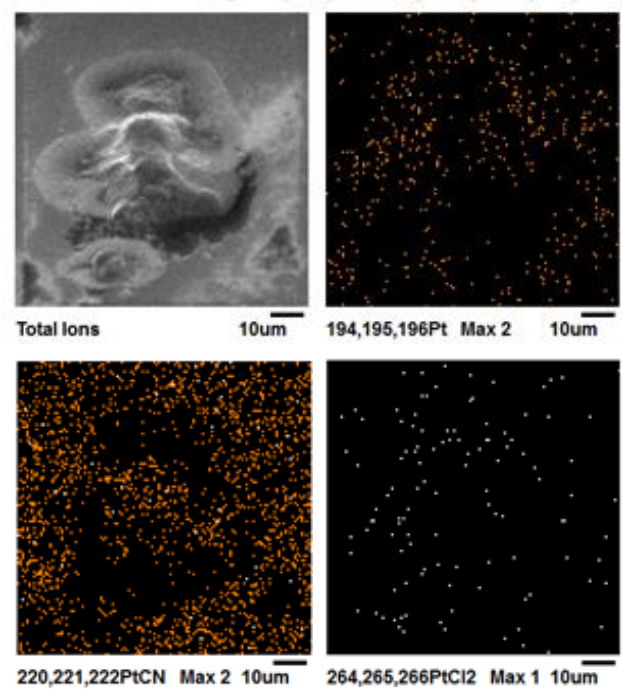

Fig. 3. Imaging of cisplatin detected from surface of cell.
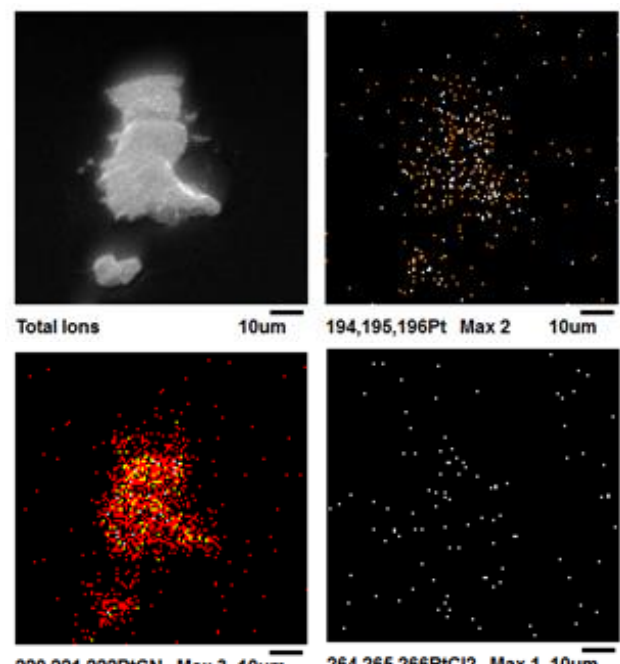

$220,221,222 \mathrm{PtCN} \operatorname{Max} 310 \mathrm{um} 264,265,266 \mathrm{PtCl} 2 \operatorname{Max} 110 \mathrm{um}$

Fig. 4. Imaging of cisplatin detected from intracellular

\section{References}

[1] T. Sakamoto, M. Koizumi, J. Kawasaki, J. Yamaguchi, Applied Surface Science, 255, 1617 (2008).

[2] M. Chikuma, T. Sato, S. Komeda, YAKUGAKU ZASSHI 128(3)307-316(2008).

\section{Acknowledgment}

This work is performed in "Joint-Research of Kogakuin University and Tokyo Medical University". Authors greatly appreciate to Prof. Ikeda, Prof. Ohira, Prof. Kajiwara, Prof. Kakihana, and Dr. Nagase (Tokyo Medical University). 